\title{
Sarin chemisorbent based on cobalt-doped graphene
}

\author{
Neeraj K. Jaiswal \\ 2-D Materials Research Laboratory, Discipline of Physics, Indian Institute of Information Technology Design $\mathcal{E}$ \\ Manufacturing, Dumna Airport Road, Jabalpur 482005, MP, India
}

Goran Kovačević*

Ruđer Bošković institute, P.O.B. 180, Bijenička 54, HR-10002 Zagreb, Croatia.

\begin{abstract}
Ion bombardment on graphene sheets can produce atomic vacancies that can trap metal atoms. In this paper, we demonstrated that these trapped metal atoms can effectively bind other molecules with heteroatoms, making them chemisorbed to the graphene. The trapped cobalt atom can bind sarin molecule through fluorine atom with dissociation energy significantly higher than the one bonded via oxygen atom. This suggests that it can displace water molecule and therefore pledge for sarin chemisorbent in atmospheric environment. Our investigations also revealed that metallic character is enhanced upon sarin adsorption unlike the bonding of water molecule with trapped metal atom in graphene lattice which causes an opening of small $(0.02 \mathrm{eV})$ band gap. Present findings can have promising application towards detecting the presence of toxic sarin molecules.
\end{abstract}

Keywords: graphene, sarin, chemisorption, density functional theory, band structure, equilibrium constant

\section{Introduction}

Many molecules with heteroatoms such as nitrogen or phosphorous, suspended in the gas phase, can be very harmful to human health if inhaled or if made contact with skin. Most notorious such compounds are chemical warfare agents that work as nerve toxins, which are harmful even in extremely low concentrations.[1] Lone electron pair on the heteroatom is available to form a donor-acceptor bond with an electron-deficient atom.[2] Since the best electron-acceptor atoms are transition metals, they can be used for chemisorption of such molecules, effectively removing them from the gas phase.[3]

Recently, it was demonstrated that atoms of cobalt can be inserted into vacancy of graphene sheet, produced by irradiation. $[4,5]$ That makes it a convenient solid medium for chemisorption with very large surface to mass ratio. All chemical bonds by which metal atoms are bonded with carbon atoms in a graphene sheet are arranged in only one plain, which leaves at least two potential binding sites, perpendicular to the plain of the graphene sheet.[4] Graphene has also the advantage of being atomically thin conductive material.[6-9] As such, any change in the electronic structure will influence its conductivity.[10, 11] That opens possibility of sensing very low concentrations of gasses.[1218] Sensor devices, made of graphene, nanotubes and other nanomaterials have been demonstrated before.[14, 16-23]

In this paper, the possibility of chemisorption of the sarin (isopropyl methylphosphonoflouridate) molecule is investigated. A great deal of research is being done on physisorption of these molecules as this is the main method for filtering them from the air.[22] The sarin is one of the most potent

\footnotetext{
*Corresponding author

Email addresses: neerajkjaiswal@gmail . com (Neeraj K. Jaiswal), gkova@irb.hr (Goran Kovačević)
} 
synthetic toxic agent, used in chemical warfare.[1,24] It has exposed fluorine and oxygen atoms, both capable of establishing covalent donor-acceptor bond.[2] In addition, binding with water molecule is researched since water molecule has similar binding capabilities as sarin and is commonly present in the air as the moisture.

\section{Theoretical methods}

Chemisorption of sarin to the immobilised cobalt atom in a graphene nanoribbon (GNR) and changes in the GNR conductivity, caused by the chemisorption was modelled with the density functional theory (DFT).[25-30]

Periodic boundary conditions were applied along all three dimensions. Dimensions of unit cells along the direction of the GNR were selected to match the dimension of the nanoribbon model in order to ensure the periodicity. Padding of at least $10 \AA$ along other two dimensions were used in order to prevent interaction of the nanoribbons between neighbouring replicas. Monkhorst-Pack scheme was used for integration in the $k$-space with the $4 \times 4 \times 1$ grid. Geometry optimisation of all models was carried out with the Perdew-Burke-Ernzerhof (PBE) functional within the generalised gradient approximation (GGA).[31] Numeric orbitals, constructed from the double zeta basis set with polarisation (DZP), were used in all geometry optimisation calculations. Spatial extension of the atomic orbitals were defined with the split-norm of 0.15 and energy shift of $250 \mathrm{meV}$. Real space grid integration was used on a grid, defined with the energy cutoff value of $200 \mathrm{Ry}$. Troullier-Martins pseudopotentials were used in all geometry optimisation calculations.[32, 33] Several conformations of the sarin molecule, bonded to the cobalt atom were used as initial geometry. Optimised conformer with the lowest energy was selected and used in further calculations. Phonon spectrum was calculated on all optimised geometries, by using the same level of theory as in geometry optimisation. Real values of resulted vibrational frequencies confirmed that all geometries correspond to energy minima. SIESTA program package was used for geometry optimisation and frequency calculations.[34]

Beside $10 \AA$ long GNR systems with immobilised cobalt atom, twice as long GNRs $(20 \AA)$ were also optimised in order to estimate the influence of periodic replicas to resulting energies and geometries. The same settings were used as with small GNRs except the smaller grid $(2 \times 2 \times 1)$ was used in $20 \AA$ long GNRs in order to achieve the same grid point separation in the $k$-space. Calculated differences in electronic contribution to dissociation energies $(0.004-0.01 \mathrm{eV}$ for sarin, and $0.07 \mathrm{eV}$ for water) were considered small enough to accept $10 \AA$ long GNR for modelling the chemisorption of sarin.

After the original geometry optimisation was performed, another run with variable cell was done. That resulted in triclinic unit cells, with axis angles insignificantly departing from $90^{\circ}$. Cells of resulting geometries were made orthorhombic (angles between unit cell axis are orthogonalised) and subjected to another fixed-cell geometry optimisation. If there was no significant rise in energy, geometries were kept in orthorhombic cells.

Electronic energy of each energy minimum was determined with single point calculations, using the Becke, three-parameter, Lee-Yang-Parr hybrid functional (B3LYP).[35, 36] The efficiency of hybrid DFT functionals is superior to the local-density-approximation (LDA) and GGA functionals when it comes to energy stability.[37, 38] B3LYP functional is a very popular choice due to its accuracy.[39, 40]

A quickstep method was used for calculations of single point energies, that uses both, gaussian orbitals and plane waves to compute the Kohn-Sham matrix and the electronic density.[41-43] Four grids were used in plane wave calculations with the energy cutoff of 250 Ry for the finest grid. Gaussian functions were constructed from the double zeta basis set with the polarisation and the Goedecker-Teter-Hutter pseudopotential (DZVP-GTH-PADE).[44, 45] Basis set superposition error was calculated by using Boys-Bernardi counterpoise correction.[46] For that purpose, calculations were repeated, but with atoms in a molecular fragment replaced with ghost atoms. The dissociation 
energy (at $0 \mathrm{~K}$ ) is then calculated from the corrected electronic energies and zero point vibrational energies:

$$
E_{d}=E_{e}^{a b}+E_{v 0}^{a b}-E_{e}^{a *}-E_{v 0}^{a}-E_{e}^{b *}-E_{v 0}^{b} ;
$$

where $E_{e}$ denotes the electronic energy (calculated with B3LYP/DZVP-GTH-PADE), $E_{v 0}$ is the vibrational zero point energy (calculated with PBE/DZP), $a b$ superscript denotes that the physical quantity refers to the dimer, superscripts $a$ or $b$ refer to monomers and superscripts $a *$ and $b *$ refer to a dimer in which one of the monomer's atoms are replaced with ghost atoms.

The effectiveness of ligand binding is expressed as standard dissociation constant.[47] For a general adsorption reaction:

$$
\mathrm{AB}(\text { surface }) \rightleftharpoons \mathrm{A}(\text { gas })+\mathrm{B}(\text { surface })
$$

where $\mathrm{A}$ is a molecule being adsorbed, $\mathrm{B}$ a bonding site on adsorbent and $\mathrm{AB}$ the molecule $\mathrm{A}$, bonded to the bonding site, standard equilibrium constant for dissociation reaction 2 can be written as:

$$
K_{d}^{\ominus}=\frac{p(\mathrm{~A}) *[\mathrm{~B}]}{[\mathrm{AB}]},
$$

where $p(\mathrm{~A})$ is partial pressure of $\mathrm{A},[\mathrm{B}]$ is surface concentration of free bonding sites and $[\mathrm{AB}]$ is surface concentration of occupied bonding sites.[47] The dissociation constant $\left(K_{d}^{\ominus}\right)$ is calculated with equation:

$$
K_{d}^{\ominus}=\left(K_{b} T\right) \mathrm{e}^{-\Delta E / K_{b} T} \prod_{i} q_{i}^{\gamma_{i}},
$$

where $K_{b}$ is the Boltzmann constant, $T$ is the temperature, $\Delta E$ is the energy difference between reactants and products, $q_{i}$ is the partition function of the $i$-th species and $v_{i}$ is its stoichiometric number. Partition functions for species in gas phase are calculated as product of translational, rotational and vibrational partition functions, assuming harmonic oscillator, rigid rotor and ideal gas approximations. In the case of solid state species, only the vibrational partition function is taken into account.

To gauge the potential of Co doped GNRs towards the detection of sarin, we examined the changes in electronic band structure due to adsorption of targeted molecule. For the computation of electronic band structures, 50 k-points along the periodic direction of the ribbon were selected. However, a densely packed mesh of $101 \mathrm{k}$ points was selected for the computation of transmission spectra of considered configurations. 


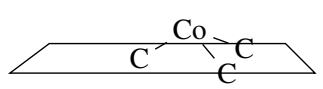

1

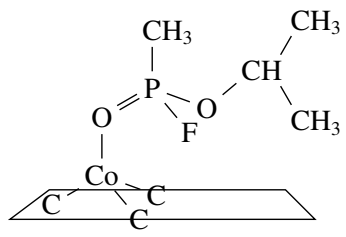

3

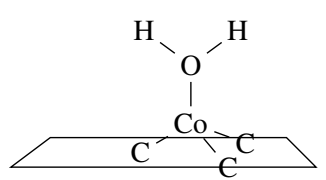

5

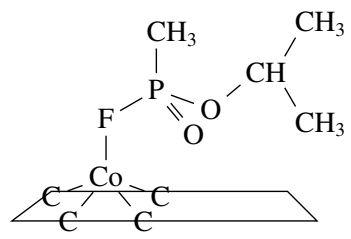

7

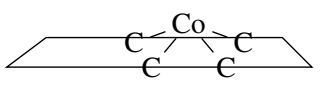

2

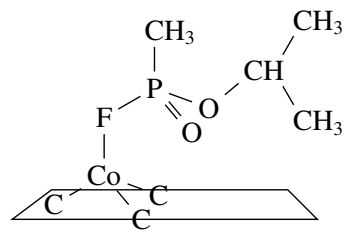

4<smiles></smiles>

6

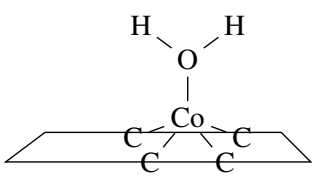

8

scheme 1: Graphene nanoribbons doped with cobalt atoms and chemisorbed sarin and water molecules.

\section{Results and discussion}

The chemisorption of the sarin molecule on GNRs, decorated with cobalt atoms (structures 1, 2), was modelled as shown in figure 1. These two structures were the result of insertion of the cobalt atom into the monovacancy (figure 1a) and divacancy (figure 1b).

Both of these structures were previously produced experimentally.[4] The cobalt atom, inserted into the monovacancy was bonded with three bonds to the GNR.

Since the monovacancy is too small to accommodate one cobalt atom, the guest (Co) atom in $\mathbf{1}$ was situated $1.2 \AA$ above the GNR plane with C-Co bond length $1.76 \AA$ and $\mathrm{C}-\mathrm{Co}-\mathrm{C}$ angle of $99^{\circ}$. The Cobalt atom in $\mathbf{2}$ is also displaced from the GNR plane, only for $0.5 \AA$. C-Co bond is longer in this case (about $1.9 \AA$ ). Four bonds around the Co atom are closing two five-membered and two six-membered rings in $\mathrm{C}_{2 \mathrm{v}}$ symmetry arrangement $\left(\mathrm{C}-\mathrm{Co}-\mathrm{C}\right.$ angles: $87^{\circ}$ and $89^{\circ}$ respectively).

Chemisorption of sarin molecules was investigated through bonding with oxygen and fluorine atoms (scheme 1). 


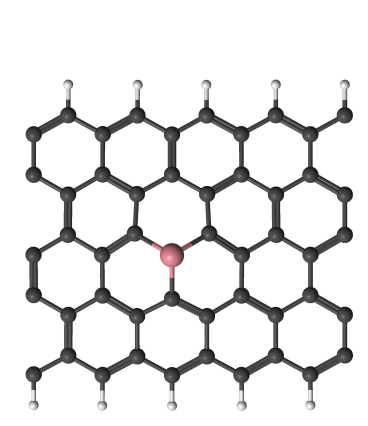

(a)

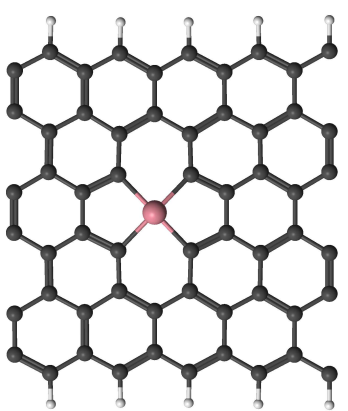

(b)

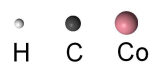

Figure 1: Graphene nanoribbons with cobalt atoms as model chemisorbents.

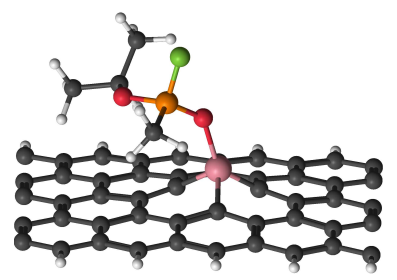

(a)

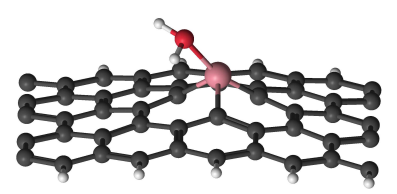

(c)

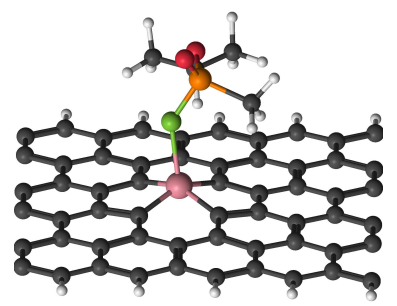

(e)

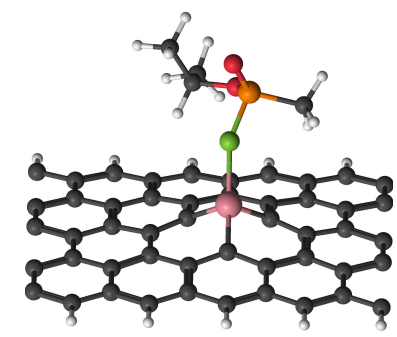

(b)

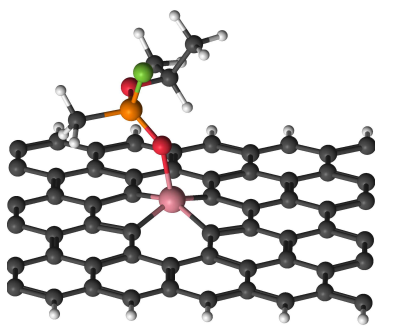

(d)

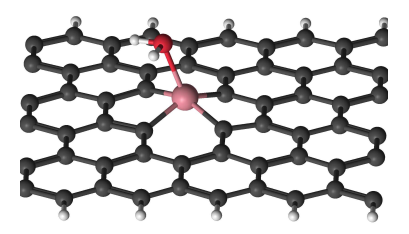

(f)

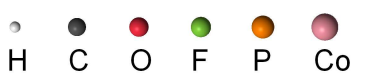

Figure 2: Chemisorbent based on graphene nanoribbons with cobalt atom in the monovacancy with: a) sarin molecule bonded with $\mathrm{O}$ atom, b) sarin molecule bonded with $\mathrm{F}$ atom, c) water molecule; and chemisorbent based on graphene nanoribbons with cobalt atom in the divacancy with: d) sarin molecule bonded with $\mathrm{O}$ atom, e) sarin molecule bonded with $\mathrm{F}$ atom, f) water molecule.

In the most stable conformers (figure 2), both organic groups (methyl and isopropyl) had contact with the GNR. Hydrogen atoms in those groups approached the plain of the GNR up to $2.3 \AA$. This conformation is similar to physisorbed sarin on graphene, determined by configuration interaction theory.[22] That contact makes additional stabilisation of the chemisorbed sarin, complementing the covalent bond with the dispersion interaction between the organic groups and the GNR. 
The possibility of sarin as multidentat ligand was also investigated. Conformations in which two (carbonyl oxygen and fluorine) and three (both oxygen and fluorine) atoms are connected to the cobalt atom were also optimised. Although these conformations have two or three covalent bonds between the sarin and the cobalt atom, the stability is not enhanced over monodentat bonding. The geometry optimisation of these structures resulted in monodentat bonded structures, shown in figure 2 .

Chemisorbed molecules were not bound to the cobalt atom perpendicularly as would be expected from tetrahedral or square pyramid coordination. The discrepancy is always larger in case of binding to $\mathbf{1}$. Also, the largest discrepancy is observed for water molecules (structures $\mathbf{5}$ and 8) $\left(41.0^{\circ}\right.$ and $\left.22.4^{\circ}\right)$. In case of oxygen-bonded sarin $\left(17.6^{\circ}\right.$ for $3,13.7^{\circ}$ for $\left.\mathbf{6}\right)$ and the fluorine-bonded sarin $\left(27.7^{\circ}\right.$ for 4 and $7.3^{\circ}$ for $\mathbf{7}$ ), the discrepancy is still significant.

Table 1: Dissociation energies $\left(E_{d}\right)$ calculated with PBE/DZP//B3LYP/DZVP-GTH-PADE and standard dissociation equilibrium constants $\left(K_{d}^{\ominus}\right)$, partition equilibrium constants at $300 \mathrm{~K}$, for sarin and water, chemisorbed on graphene nanoribbon containing cobalt atoms $\left(K_{D}\right)$.

\begin{tabular}{llll}
\hline system & $E_{d} / \mathrm{eV}$ & $K_{d}^{\ominus} / \mathrm{Pa}$ & $K_{D}$ \\
\hline $\mathbf{3}$ & 1.08 & $1.07 \times 10^{-25}$ & 46729 \\
$\mathbf{4}$ & 1.24 & $2.21 \times 10^{-28}$ & $2.26 \times 10^{+7}$ \\
$\mathbf{5}$ & 0.48 & $5.00 \times 10^{-21}$ & - \\
$\mathbf{6}$ & 0.46 & $2.90 \times 10^{-15}$ & $2.39 \times 10^{-5}$ \\
$\mathbf{7}$ & -0.57 & 309.85 & $2.24 \times 10^{-22}$ \\
$\mathbf{8}$ & 0.41 & $6.93 \times 10^{-20}$ & - \\
\hline
\end{tabular}

Almost all structures show positive dissociation energies (table 1), that are significantly larger than the thermal energy at room temperature. The only case with negative dissociation energy is 7 . Also, sarin bonds with 1 stronger than with $\mathbf{2}$. In $\mathbf{1}$, cobalt atom is in Co ${ }^{\mathrm{III}}$ oxidation state, which is much more common than $\mathrm{Co}^{\mathrm{IV}}$ (as in 2).[2] The stabilisation upon binding sarin (or water), can be attributed to increase in coordination to 4 , which is, not most common coordination for $\mathrm{Co}^{\mathrm{III}}$ complexes.[2] The pyramidal coordination in 6-8 is rather unusual in cobalt chemistry, especially for $\mathrm{Co}^{\mathrm{IV}}$ atom.[2]

Zero point vibrational energy (ZPVE) corrections are very small compared to total dissociation energies, however they are not uniform. The greatest correction is for binding of water molecules to both: $1(-0.061 \mathrm{eV})$ and $2(-0.04 \mathrm{eV})$. ZPVE corrections for bonding of sarin to 2 increases the dissociation energy (makes complex less stable) for $0.01 \mathrm{eV}$ (O-bonding) and $0.039 \mathrm{eV}$ (F-bonding), while binding to 1 makes insignificant corrections (relative to thermal energy): $-0.0002 \mathrm{eV}$ (O-bonding) and $0.007 \mathrm{eV}$ (F-bonding).

Energy alone is not the only factor that to affect the effectiveness of adsorption. Bonding of a molecule to a stationary phase makes several translational and rotational degrees of freedom disappear. Since these degrees of freedom carry a considerable amount of entropy, adsorption in general is unfavourable process, unless the adsorption enthalpy is sufficiently negative or there is another source of entropy in adsorbed species.[47] The effectiveness of adsorption can be much better described by the equilibrium constant. Table 1 shows dissociation equilibrium constants for compounds $\mathbf{3}-\mathbf{8}$. Most of the $K_{d}^{\ominus}$ values are significantly lower than 1, indicating that under equilibrium conditions, with enough binding sites on the cobalt-doped graphene surface, partial pressure of sarin will be reduced to essentially zero. The equilibrium constant for dissociation of $\mathbf{4}$ is several orders of magnitude lower than for $\mathbf{3}$ or $\mathbf{5}$.

Since only one bond is being broken during the dissociation of a complex, it can be assumed that reactions, represented by equation 2 have no transition states. In that case reaction barriers are equal to reaction dissociation energies, which makes reaction rates proportional to reaction equilibrium constants $\left(K_{d}^{\ominus}\right)$. That convenience can be utilised to calculate dissociation half-times $(\tau)$, which can show if an adsorbent can self-recover in a reasonable time. Transition state theory, first order reaction kinetics and all transmission coefficients equal to one (no dissociated molecule, binds back to the 
adsorbent) are assumed in recovery time $(\tau)$ calculation with equation 5:

$$
\tau=\frac{\ln (2) h}{K_{b} T K_{d}^{\ominus}},
$$

where $h$ is the Planck constant. Complexes with largest dissociation energies ( $\mathbf{3}$ and $\mathbf{4}$ ) have extremely long dissociation times $\left(1.65 \times 10^{11} \mathrm{~s}\right.$ and $\left.8.00 \times 10^{13} \mathrm{~s}\right)$ making adsorption practically irreversible at room temperature. Water dissociates in days $\left(\mathbf{8}, 2.55 \times 10^{5} \mathrm{~s}\right)$ to months $\left(\mathbf{5}, 3.53 \times 10^{6} \mathrm{~s}\right)$, while only $\mathbf{6}$ dissociates in a time span ( $6.09 \mathrm{~s})$, convenient for a self-regenerating sensor. Multiple-usage sensors, based on $\mathbf{1}$ and $\mathbf{2}$ would require assisted desorption by UV irradiation or electric field.[48, 49]

The preference of $\mathbf{1}$ for binding with sarin over water can partially attributed to the additional non-bonded interactions. It was demonstrated that non-bonded interactions in metal-organic frameworks (MOF) with hydrophobic cavities, can be used for selectively capturing molecules similar to sarin (Diisopropylfluorophosphate, DIFP).[50] In the same paper, it was found that the MOF that contains coordinatively unsaturated metal centre $\left(\left[\mathrm{Cu}_{3}(\mathrm{btc})_{2}\right]\right)$ binds water with similar affinity as DIFP.[50] Unlike copper complexes[50], there is a very strong selectivity towards sarin over water molecule in 1. Unfortunately, $\mathbf{2}$ shows opposite behaviour: partition constants for sarin/water on $\mathbf{2}$ (table 1) are significantly smaller than 1 . Dissociation energies of sarin and water with $\mathbf{2}$ is of similar magnitude with measured heats of adsorption with $\left[\mathrm{Cu}_{3}(\mathrm{btc})_{2}\right]\left(\Delta H_{a d s}(\mathrm{DIFP})=48.4 \mathrm{~kJ} / \mathrm{mol}=\right.$ $\left.0.50 \mathrm{eV} ; \Delta H_{a d s}\left(\mathrm{H}_{2} \mathrm{O}\right)=43.9 \mathrm{~kJ} / \mathrm{mol}=0.45 \mathrm{eV}\right)$. However, there are significant differences in binding between MOFs and metal-doped graphene. In MOFs, molecules are adsorbed on surfaces of tiny cavities inside structures of MOFs, rather than on a single flat surface. Also, DIFP lacks phosphoryl oxygen atom $(\mathrm{P}=\mathrm{O})$. In all optimised geometries, cobalt atom forms bond with the phosphoryl oxygen, rather with the alkyl oxygen atom. This is in agreement with sarin adsorption on anatas surface[51], where it was demonstrated that the phosphoryl oxygen is preferred for bonding. Experimental evidence show that organophosphonate compounds prefer binding to surfaces containing hydroxyl groups or metal atoms.[52] While binding to hydroxyl groups involves different mechanism than described here (hydrogen bonding), change in IR frequencies of phosphoryl oxygen upon sorption demonstrated type of bonding described here.[52]

The importance of Co doping to bind the sarin molecule with graphene is further evident while comparing the present findings with those of Papas et.al.[22] Their findings based on configuration interaction as well as DFT calculations revealed a weaker bonding between sarin and perfect graphene as compared to present results with Co impurity in graphitic network. In another study, adsorption of sarin on BN has been reported by considering its sheet, nanotube and nanocage configurations.[53] Here also, doping of various impurities were found to enhance the binding of sarin which is consistent to our observations with Co impurity in graphene. Further, their calculated adsorption energy (-13.8 $\mathrm{kcal} / \mathrm{mol}$ ) is in excellent agreement with our reported value of $-0.57 \mathrm{eV}$ (i.e. $-13.14 \mathrm{kcal} / \mathrm{mol}$ ). 


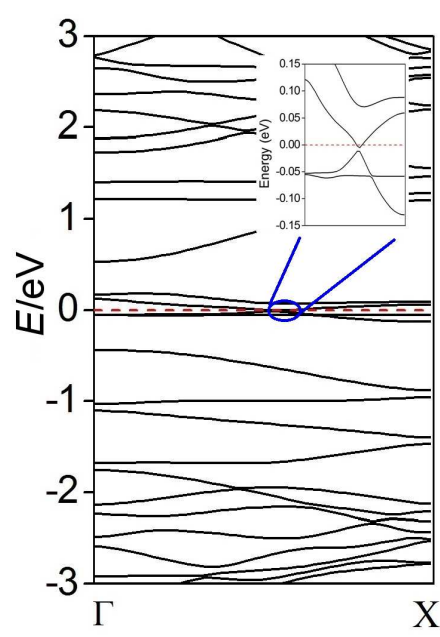

(a)

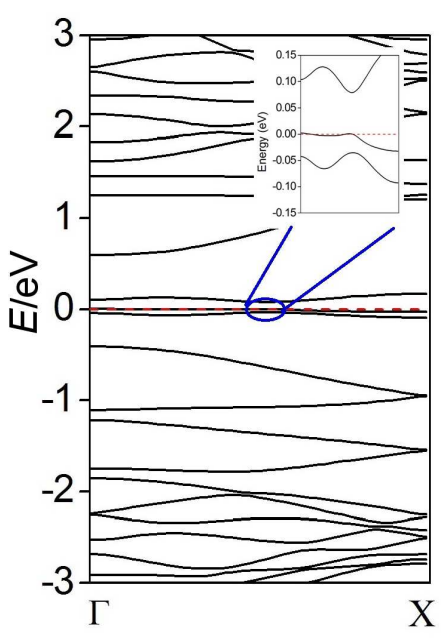

(b)

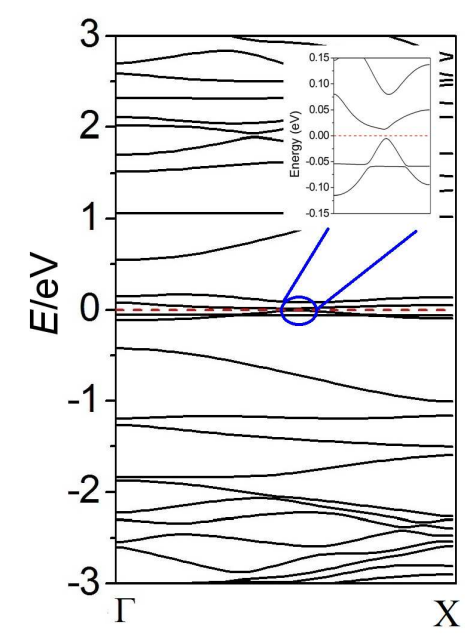

(c)

Figure 3: Band structures of graphene nanoribbons, doped with cobalt. (a) No ligand; (b) sarin bound through fluorine atom to the cobalt atom; (c) water molecule bound to the cobalt atom.

When sarin bonds with $\mathbf{1}$ via oxygen atom (compound $\mathbf{3}$ ), it will rebond into thermodynamically more favourable $\mathbf{4}$. More important is the ability of sarin to displace water from $\mathbf{5}$. A practical adsorbent should be able to bind targeted molecule regardless of presence of competitive molecules in the atmosphere. Water molecule, as a common ligand in $\mathrm{Co}^{\mathrm{III}}$ chemistry, is also common in atmosphere as moisture and exposing the adsorbent to air will be, given enough time, saturated with water. The difference in dissociation equilibrium constants guarantee that sarin will displace water on the adsorbent. Data in table 1 show that $\mathbf{2}$ does not perform as a good adsorbent, as dissociation energy (and therefore corresponding equilibrium constant) of the most stable form of bonded sarin (6) is significantly lower than the corresponding structure in the case of $\mathbf{1}$. Also, $\mathbf{7}$ shows negative dissociation energy indicating it is less stable than reactants. Although there is possibility that it exists as metastable species (if there exists a corresponding transition state with higher energy than 7), its existence is beyond the scope of this paper as it would anyway fail as adsorbent. Moreover, 2 binds water (8) so strongly, that it would not be able to displace it with sarin. Therefore electronic properties of $\mathbf{2}$ will not be considered due to its poor adsorbing performance.

The perusal of electronic band structures (figure 3) for Co doped GNR reveals that electronic bands are lying in the vicinity of Fermi level. However, a magnified view of the same, confirms that lowest conduction band (LCB) is merely touching the Fermi level (figure 3a). Therefore, it suggests for the semi-metallic character of this structure. On the other hand, the highest valence band (HVB) and LCB are moving apart as a result of sarin adsorption and an additional dispersive band appears in between and across the Fermi level (figure 3b). This additional dispersive band suggests for the enhanced metallicity as compared to Co doped GNR. Interestingly, the adsorption of $\mathrm{H}_{2} \mathrm{O}$ molecule opens up a small (direct) band gap across the Fermi level. The magnitude of this band gap is found to be $0.02 \mathrm{eV}$ in the middle of $\Gamma$ to $\mathrm{X}$. To further explore the effect of sarin adsorption on transport properties, the transmission spectra (TS) have been plotted for all three configurations as shown in figure 4. From TS profiles, a sharp peak lying across the Fermi level (figure $4 b$ ) can be distinctly identified in Co doped GNR under the influence of sarin adsorption.

However, in the absence of sarin molecule, we observed a rather blunt peak for Co doped GNR with reduced magnitude which is supporting its semi-metallic character. Finally, the absence of transmission channel (at the Fermi level) has been noticed for $\mathrm{H}_{2} \mathrm{O}$ absorbed configuration as shown in figure $4 \mathrm{c}$ which is due to the opening of small band gap for corresponding band structure (figure $3 \mathrm{c}$ ). 


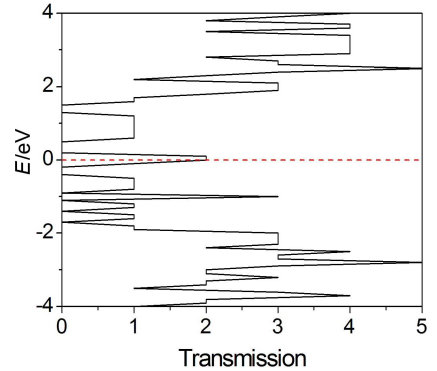

(a)

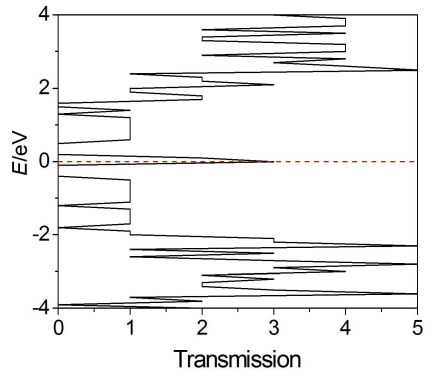

(b)

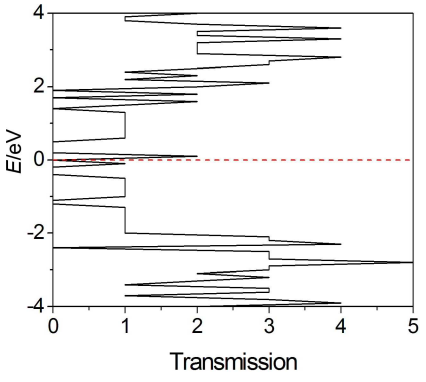

(c)

Figure 4: Transmission spectra for graphene nanoribbon, doped with cobalt. (a) No ligand; (b) sarin bound through fluorine atom to the cobalt atom; (c) water molecule bound to the cobalt atom.

\section{Conclusion}

Graphene with induced monovacancies and doped with cobalt atoms most efficiently bind sarin molecules through fluorine atoms. Calculated dissociation energies and dissociation equilibrium constants indicate that sarin binds to the cobalt-doped graphene more strongly than water, which makes it practical chemisorbent as sarin can displace bonded water. Cobalt-doped graphene with induced divacancies proved to be poor chemisorbent for sarin with very strong binding to water. Binding of sarin to the cobalt atom in graphene monovacancies, closes its band gap, enhancing its metallicity. Since the water molecule, bonded with the cobalt atom induces very small band gap, there is a significant change in the electronic structure upon binding different molecules to use this material as a sarin gas sensor.

\section{Contributions}

GK did geometry optimisation and single point energy calculations and written the corresponding part of the manuscript; NKJ made electronic properties calculations and wrote the corresponding part of the manuscript.

\section{Acknowledgements}

Some calculations were performed on equipment donated by Zagreb University Computing Centre (Srce). NKJ is thankful to DST-SERB for providing computational facility under the DST Young Scientist Research grant SB/FTP/PS-101/2014.

[1] L. S. Goodman, The pharmacological basis of therapeutics, 5th Edition, MacMillan, New York, cop. 1975.

[2] F. A. Cotton, G. Wilkinson, Advanced inorganic chemistry, 5th Edition, John Wiley, New York, 1988.

[3] E. A. Tutov, V. P. Zlomanov, Effect of chemisorption of donor and acceptor gases on the semiconductor-metal phase transition in vanadium dioxide films, Phys. Solid State 55 (11) (2013) 2351-2354.

[4] H. Wang, Q. Wang, Y. Cheng, K. Li, Y. Yao, Q. Zhang, C. Dong, P. Wang, U. Schwingenschlögl, W. Yang, X. X. Zhang, Doping monolayer graphene with single atom substitutions, Nano Lett. 12 (1) (2012) 141-144. 
[5] H. Vázquez, E. Åhlgren, O. Ochedowski, A. Leino, R. Mirzayev, R. Kozubek, H. Lebius, M. Karlušic, M. Jakšic, A. Krasheninnikov, J. Kotakoski, M. Schleberger, K. Nordlund, F. Djurabekova, Creating nanoporous graphene with swift heavy ions, Carbon 114 (2017) 511 518.

[6] K. S. Novoselov, A. K. Geim, S. V. Morozov, D. Jiang, Y. Zhang, S. V. Dubonos, I. V. Grigorieva, A. A. Firsov, Electric field effect in atomically thin carbon films, Science 306 (5696) (2004) 666-669.

[7] K. S. Novoselov, D. Jiang, F. Schedin, T. J. Booth, V. V. Khotkevich, S. V. Morozov, A. K. Geim, Two-dimensional atomic crystals, Proc. Natl. Acad. Sci. U.S.A 102 (30) (2005) 10451-10453.

[8] L. Wirtz, A. Rubio, The phonon dispersion of graphite revisited, Solid State Commun. 131 (3) (2004) $141-152$.

[9] L. Malard, M. Pimenta, G. Dresselhaus, M. Dresselhaus, Raman spectroscopy in graphene, Phys. Reports 473 (5) (2009) $51-87$.

[10] V. P. Gusynin, S. G. Sharapov, J. P. Carbotte, Ac conductivity of graphene: From tight-binding model to 2 + 1-dimensional quantum electrodynamics, Int. J. Modern Phy. B 21 (27) (2007) 4611-4658.

[11] W. Qin, X. Li, W.-W. Bian, X.-J. Fan, J.-Y. Qi, Density functional theory calculations and molecular dynamics simulations of the adsorption of biomolecules on graphene surfaces, Biomaterials 31 (5) (2010) $1007-1016$.

[12] F. Schedin, A. K. Geim, S. V. Morozov, E. W. Hill, P. Blake, M. I. Katsnelson, K. S. Novoselov, Detection of individual gas molecules adsorbed on graphene, Nature Mater. 6 (2007) 652-655.

[13] H. E. Romero, P. Joshi, A. K. Gupta, H. R. Gutierrez, M. W. Cole, S. A. Tadigadapa, P. C. Eklund, Adsorption of ammonia on graphene, Nanotechnol. 20 (24) (2009) 245501.

[14] M. Gautam, A. H. Jayatissa, Adsorption kinetics of ammonia sensing by graphene films decorated with platinum nanoparticles, J. Appl. Phys. 111 (9) (2012) 094317.

[15] S. Bai, Y. Zhao, J. Sun, Y. Tian, R. Luo, D. Li, A. Chen, Ultrasensitive room temperature nh3 sensor based on a graphene-polyaniline hybrid loaded on pet thin film, Chem. Commun. 51 (2015) 7524-7527.

[16] M. Kościński, M. Seredych, T. J. Bandosz, M. M. Śliwińska Bartkowiak, Electrical characterization of ammonia carbon-based sensors, Acta Phys. Pol. A 128 (2015) 182-184.

[17] Z. Zhang, X. Zhang, W. Luo, H. Yang, Y. He, Y. Liu, X. Zhang, G. Peng, Study on adsorption and desorption of ammonia on graphene, Nanoscale Res. Lett. 10 (1) (2015) 1-8.

[18] A. Zhang, Y. Wu, B. Yao, Y. Gong, Optimization study on graphene-coated microfiber bragg grating structures for ammonia gas sensing, Photonic Sensors 5 (1) (2015) 84-90.

[19] R. Chowdhury, S. Adhikari, P. Rees, S. P. Wilks, F. Scarpa, Graphene-based biosensor using transport properties, Phys. Rev. B 83 (2011) 045401.

[20] E. Ashori, F. Nazari, F. Illas, Adsorption of H2S on carbonaceous materials of different dimensionality, Int. J. Hydrogen Energy 39 (12) (2014) 6610-6619. 
[21] Z. Chen, P. Darancet, L. Wang, A. C. Crowther, Y. Gao, C. R. Dean, T. Taniguchi, K. Watanabe, J. Hone, C. A. Marianetti, L. E. Brus, Physical Adsorption and Charge Transfer of Molecular Br2 on Graphene, ACS Nano 8 (3) (2014) 2943-2950.

[22] B. N. Papas, I. D. Petsalakis, G. Theodorakopoulos, J. L. Whitten, CI and DFT Studies of the Adsorption of the Nerve Agent Sarin on Surfaces, J. Phys. Chem. C 118 (40) (2014) 2304223048.

[23] Y. Wang, L. Zhang, N. Hu, Y. Wang, Y. Zhang, Z. Zhou, Y. Liu, S. Shen, C. Peng, Ammonia gas sensors based on chemically reduced graphene oxide sheets self-assembled on au electrodes, Nanoscale Res. Lett. 9 (1) (2014) 251.

[24] T.-M. Shih, J. H. McDonough, Efficacy of biperiden and atropine as anticonvulsant treatment for organophosphorus nerve agent intoxication, Arch. Toxicol. 74 (3) (2000) 165-172.

[25] P. Hohenberg, W. Kohn, Inhomogeneous electron gas, Phys. Rev. 136 (1964) B864-B871.

[26] Ceperley, D. M., Alder, B. J., The low density phases of the electron gas, J. Phys. Colloq. 41 (C7) (1980) C7-295-C7-298.

[27] R. O. Jones, O. Gunnarsson, The density functional formalism, its applications and prospects, Rev. Mod. Phys. 61 (1989) 689-746.

[28] E. J. Baerends, O. V. Gritsenko, A quantum chemical view of density functional theory, J. Phys. Chem. A 101 (30) (1997) 5383-5403.

[29] J. P. Perdew, A. Ruzsinszky, L. A. Constantin, J. Sun, G. I. Csonka, Some fundamental issues in ground-state density functional theory: A guide for the perplexed, J. Chem. Theory Comput. 5 (4) (2009) 902-908.

[30] P. J. Hasnip, K. Refson, M. I. J. Probert, J. R. Yates, S. J. Clark, C. J. Pickard, Density functional theory in the solid state, Philos. Trans. R. Soc. London, Ser. A 372 (2011) (2014) 20130270.

[31] J. P. Perdew, K. Burke, M. Ernzerhof, Generalized gradient approximation made simple, Phys. Rev. Lett. 77 (1996) 3865-3868.

[32] G. P. Kerker, Non-singular atomic pseudopotentials for solid state applications, J. Phys. C: Solid State Phys. 13 (9) (1980) L189.

[33] N. Troullier, J. L. Martins, Efficient pseudopotentials for plane-wave calculations, Phys. Rev. B 43 (1991) 1993-2006.

[34] J. M. Soler, E. Artacho, J. D. Gale, A. García, J. Junquera, P. Ordejón, D. Sánchez-Portal, The siesta method for ab initio order- $\mathrm{n}$ materials simulation, J. Phys.: Condens. Matter 14 (11) (2002) 2745.

[35] C. Lee, W. Yang, R. G. Parr, Development of the colle-salvetti correlation-energy formula into a functional of the electron density, Phys. Rev. B 37 (1988) 785-789.

[36] A. D. Becke, Density-functional thermochemistry. iii. the role of exact exchange, J. Chem. Phys. 98 (7) (1993) 5648-5652.

[37] J. P. Perdew, A. Ruzsinszky, J. Tao, V. N. Staroverov, G. E. Scuseria, G. I. Csonka, Prescription for the design and selection of density functional approximations: More constraint satisfaction with fewer fits, J. Chem. Phys. 123 (6) (2005) 062201. 
[38] A. Cohen, P. Mori-Sánchez, W. Yang, Insights into current limitations of density functional theory, Science 321 (5890) (2008) 792-794.

[39] S. Hammes-Schiffer, A conundrum for density functional theory, Science 355 (6320) (2017) $28-29$.

[40] M. G. Medvedev, I. S. Bushmarinov, J. Sun, J. P. Perdew, K. A. Lyssenko, Density functional theory is straying from the path toward the exact functional, Science 355 (6320) (2017) 49-52.

[41] B. G. Lippert, J. Hutter, M. Parrinello, A hybrid gaussian and plane wave density functional scheme, Mol. Phys. 92 (3) (1997) 477-488.

[42] G. Lippert, J. Hutter, M. Parrinello, The gaussian and augmented-plane-wave density functional method for ab initio molecular dynamics simulations, Theor. Chem. Acc. 103 (2) (1999) 124 140 .

[43] J. VandeVondele, M. Krack, F. Mohamed, M. Parrinello, T. Chassaing, J. Hutter, Quickstep: Fast and accurate density functional calculations using a mixed gaussian and plane waves approach, Comput. Phys. Commun. 167 (2) (2005) 103 - 128.

[44] S. Goedecker, M. Teter, J. Hutter, Separable dual-space gaussian pseudopotentials, Phys. Rev. B 54 (1996) 1703-1710.

[45] C. Hartwigsen, S. Goedecker, J. Hutter, Relativistic separable dual-space gaussian pseudopotentials from h to rn, Phys. Rev. B 58 (1998) 3641-3662.

[46] S. Boys, F. Bernardi, The calculation of small molecular interactions by the differences of separate total energies. some procedures with reduced errors, Mol. Phys. 19 (4) (1970) 553-566.

[47] P. W. Atkins, Physical chemistry, 6th Edition, Oxford university press, Oxford, 1998.

[48] R. J. Chen, N. R. Franklin, J. Kong, J. Cao, T. W. Tombler, Y. Zhang, H. Dai, Molecular photodesorption from single-walled carbon nanotubes, Appl. Phys. Lett. 79 (14) (2001) 2258-2260.

[49] M. P. Hyman, J. W. Medlin, Theoretical study of the adsorption and dissociation of oxygen on pt(111) in the presence of homogeneous electric fields, J. Phys. Chem. B 109 (13) (2005) 6304-6310.

[50] C. Montoro, F. Linares, E. Quartapelle Procopio, I. Senkovska, S. Kaskel, S. Galli, N. Masciocchi, E. Barea, J. A. R. Navarro, Capture of nerve agents and mustard gas analogues by hydrophobic robust mof-5 type metal-organic frameworks, J. Am. Chem. Soc. 133 (31) (2011) 11888-11891.

[51] N. Q. Le, C. E. Ekuma, B. I. Dunlap, D. Gunlycke, First-principles calculations of sarin adsorption on anatase surfaces, J. Phys. Chem. C 122 (5) (2018) 2832-2839.

[52] K. Kim, O. G. Tsay, D. A. Atwood, D. G. Churchill, Destruction and detection of chemical warfare agents, Chem. Rev. 111 (9) (2011) 5345-5403.

[53] R. Amirkhani, M. H. Omidi, R. Abdollahi, H. Soleymanabadi, Investigation of sarin nerve agent adsorption behavior on bn nanostructures: Dft study, J. Cluster Sci. 29 (4) (2018) 757-765. 